\title{
Performance of serum-supplemented and serum-free media in IFN $\gamma$ Elispot Assays for human T cells
}

\author{
Sylvia Janetzki $\cdot$ L. Price $\cdot$ C. M. Britten $\cdot$ S. H. van der Burg $\cdot$ J. Caterini $\cdot$ \\ J. R. Currier · G. Ferrari · C. Gouttefangeas $\cdot$ P. Hayes $\cdot$ E. Kaempgen $\cdot$ \\ V. Lennerz $\cdot$ K. Nihlmark $\cdot$ V. Souza $\cdot$ A. Hoos
}

Received: 9 September 2009 / Accepted: 16 October 2009 / Published online: 6 November 2009

(c) The Author(s) 2009. This article is published with open access at Springerlink.com

\begin{abstract}
The choice of serum for supplementation of media for $\mathrm{T}$ cell assays and in particular, Elispot has been a major challenge for assay performance, standardization, optimization, and reproducibility. The Assay Working Group of the Cancer Vaccine Consortium (CVC-CRI) has recently identified the choice of serum to be the leading cause for variability and suboptimal performance in large international Elispot proficiency panels. Therefore, a serum task force was initiated to compare the performance of commercially available serum-free media to laboratories'
\end{abstract}

S. Janetzki · A. Hoos

Cancer Vaccine Consortium of the Cancer Research Institute,

New York, NY, USA

S. Janetzki $(\bowtie)$

ZellNet Consulting, Inc, 555 North Avenue,

Suite 25-S, Fort Lee, NJ 07024, USA

e-mail: sylvia@zellnet.com

L. Price

Department of Biostatistics,

New York University Medical Center, New York, NY, USA

\section{M. Britten}

Division of Experimental and Translational Oncology,

Department of Internal Medicine III,

Johannes Gutenberg-University, Mainz, Germany

S. H. van der Burg

Department of Clinical Oncology,

Leiden University Medical Center,

Leiden, The Netherlands

J. Caterini

sanofi pasteur, Toronto, Canada

J. R. Currier

Henry M. Jackson Foundation, Rockville, MD, USA own medium/serum combinations. The objective of this project was to investigate whether a serum-free medium exists that performs as well as lab-own serum/media combinations with regard to antigen-specific responses and background reactivity in Elispot. In this way, a straightforward solution could be provided to address the serum challenge. Eleven laboratories tested peripheral blood mononuclear cells (PBMC) from four donors for their reactivity against two peptide pools, following their own Standard Operating Procedure (SOP). Each laboratory

\author{
G. Ferrari \\ Department of Surgical Sciences, \\ Duke University Medical Center, Durham, NC, USA \\ C. Gouttefangeas \\ Department of Immunology, Institute for Cell Biology, \\ Eberhard-Karls University, Tübingen, Germany \\ P. Hayes \\ IAVI Human Immunology Laboratory, \\ Imperial College, London, UK \\ E. Kaempgen \\ Department of Dermatology, \\ University Hospital Erlangen, Erlangen, Germany \\ V. Lennerz \\ Department of Internal Medicine III, \\ Johannes Gutenberg-University, Mainz, Germany \\ K. Nihlmark \\ Mabtech AB, Nacka Strand, Sweden \\ V. Souza \\ Wyeth Research, Pearl River, NY, USA \\ A. Hoos \\ Bristol-Myers Squibb, Wallingford, CT, USA
}


performed five simultaneous experiments with the same SOP, the only difference between the experiments was the medium used. The five media were lab-own serum-supplemented medium, AIM-V, CTL, Optmizer, and X-Vivo. The serum task force results demonstrate compellingly that serum-free media perform as well as qualified medium/ serum combinations, independent of the applied SOP. Recovery and viability of cells are largely unaffected by serum-free conditions even after overnight resting. Furthermore, one serum-free medium was identified that appears to enhance antigen-specific IFN $\gamma$-secretion.

Keywords Elispot · Serum · Immune monitoring · Harmonization

\section{Introduction}

Immune monitoring is gaining an increasingly important role in the developmental pipeline of immuno-therapeutical vaccines including patient assessment for early immune responses after vaccination [1]. New immune monitoring techniques are constantly introduced, and known techniques are adapted and further developed to meet the demands of "exploring it all" [2, 3]. Nevertheless, a large focus has been placed on the evaluation of $\mathrm{T}$ cell responses with well-established assays like Elispot, intracellular cytokine staining (ICS) and HLA-peptide multimer staining. Elispot has earned its place as an excellent screening tool due to its outstanding sensitivity and robustness, allowing the detection of antigen-specific $\mathrm{T}$ cells in frequencies even as low as $0.001 \%$ [4]. For patient assessment, many translational and clinical laboratories optimize and standardize their Elispot protocol, which will be eventually exposed to qualification and validation procedures [5-7]. Since no gold standard exists for this bioassay, participation in specifically designed proficiency panels is a prerequisite for checking assay performance and provides a point of comparison to other laboratories testing the same samples for the same reactivity, following their lab-own protocol $[8,9]$. In 2005, the CVC-CRI addressed this need by initiating a proficiency panel program for Elispot, followed by programs for ICS and HLA-peptide multimer staining [10, 11]. The program goal is to provide an external quality assurance program and to harmonize the assay by identifying crucial protocol variables that critically influence assay performance. This offers an elegant solution for assay optimization without imposing one standardized protocol on individual laboratories. As a results of the first two Elispot proficiency panels, initial harmonization guidelines for Elispot [10] were developed. These guidelines included the need for laboratories to pretest their serum for optimal signal to noise ratio. In fact, the choice of serum was singled out as the leading cause for sub-optimal Elispot performance and variability in the first two panels, as it was in follow-up panels ([10], Janetzki unpublished).

While the influence of serum on $\mathrm{T}$ cell reactivity is known to $\mathrm{T}$ cell immunologists, there are very few published reports that address this issue in context of immune monitoring $[6,12,13]$. Other reports focus on optimal medium conditions for obtaining well-matured or clinical grade dendritic cells [14-17], identifying serum-free conditions with various supplements as an optimal choice. Interestingly, Martunizzi et al. [13] found AIM-V serum-free medium supplemented with low doses of IL-7 to perform better in Elispot compared to own, human serum-supplemented media.

Moreover, it is a "known" secret that batches from the same manufacturer or even the same serum can differ widely in performance due to non-specific activation of $\mathrm{T}$ cells or suppression of antigen-specific responses. Thus, many laboratories pretest multiple sera in order to identify one suitable batch. Once such serum has been identified, laboratories typically purchase the entire lot, in order to guarantee the same performance for long-term studies. However, these laboratories are still affected by the serum's shelf life.

In order to address the difficulties posed by the serum used in Elispot assays, the CVC-CRI Assay Working group formed a serum task force with 11 consistently high performing laboratories from the CVC panel as well as other collaborating organizations (CHAVI, C-IMT-CIP, HVTN, and IAVI). The objective of this project was to determine whether a serum-free medium exists that performs equally well in Elispot compared with serum-supplemented media from different laboratories, independent of serum type or batch, and independent of the SOP applied. Here, we describe the results of this project with specific attention to antigen-specific spots and background reactivity as well as recovery and viability after thawing and after overnight resting. Spot sizes were also assessed in response to multiple reports from serum task force members about obvious medium-specific size differences observed during plate evaluation.

\section{Materials and methods}

\section{Serum task force participants}

Eleven laboratories from the $\mathrm{CVC}$ and collaborating organizations (CHAVI, C-IMT-CIP, HVTN, and IAVI) participated. These labs represent academia, the biotech/ pharmaceutical sector, Elispot reagent manufacturer, and the US Department of Defense. All labs were identified as high-performing, experienced laboratories with Elispot 
SOPs in place. All but one lab used serum-supplemented media; one lab used AIM-V without serum supplementation, based on pretesting experiments.

\section{PBMC and antigens}

PBMC were obtained from the Immunology Quality Assurance Center Laboratory (IQAC) of the Duke Human Vaccine Institute, a division of the Duke University Medical Center in Durham NC. For this effort, the IQAC laboratory collected PBMC samples from four healthy, HIV-1/2 sero-negative human volunteers via leukapheresis. Volunteers were recruited through IRB-approved methods. Leukopaks were processed fresh in the IQAC laboratory, within $4 \mathrm{~h}$ of collection. Separation of PBMC was accomplished by density gradient centrifugation. PBMC were cryo-preserved in 10\% DMSO and 90\% heat-inactivated FBS at 15 Million cells per vial using an automated controlled rate freezer. Cryo-preserved PBMC were then transferred and stored in two vapor phase LN2 freezers for sample redundancy.

This study utilized the CEF and CMV pp65 control peptide pools as antigen sources. While the CEF peptide pool, obtained through the NIH AIDS Research and Reference Reagent Program, Division of AIDS, NIAID, NIH, contains 32 8-11mers known to elicit CD8-restricted responses [18], the CMV pp65 peptide pool, which was a generous gift of the NIAID and Becton Dickinson, consists of 135 15 mers overlapping by 11 amino acids and elicits CD8- and CD4-restricted responses [19]. The above donors exhibited different response levels against both peptide pools, and obtained PBMC were Elispot-tested at IQAC before shipping. PBMC and peptides were shipped in liquid nitrogen dry shippers using IATA approved methods. All procedures were done in GCLP compliance.

\section{Media}

Each lab used their own medium/serum combination based on previous pretesting results (Table 1). Further, laboratories obtained the following media for comparative analysis from their country-specific provider, thus each lab used the same media, but likely from different production lots:

1. AIM-V therapeutic grade (Invitrogen);

2. CTL Wash and Test medium (Cellular Technology Ltd.);

3. Optmizer T-cell expansion SFM (Invitrogen);

4. X-Vivo 15 (Lonza, Fisher Scientific).

No addition of any serum or other additives was allowed to any of the serum-free media, for any protocol step.
Table 1 Medium/serum composition and serum provider among serum task force laboratories

\begin{tabular}{lll}
\hline Lab location & Lab medium/serum & Serum provider \\
\hline Canada & RPMI/HuS & Valley Biomedical \\
Germany & IMDM/HuS & cc-pro \\
Germany & RPMI/HuS & Lonza \\
Germany & RPMI/HuS & Cambrex \\
Germany & AIM-V & - \\
Sweden & RPMI/FBS & Gibco/Invitrogen \\
UK & RPMI/FCS & Sigma \\
USA & RPMI/FCS & Atlanta Biologics \\
USA & NHS/HuS & GemCell \\
USA & RPMI/FBS & GemBio \\
The Netherlands & IMDM/FCS & PAA Laboratories \\
\hline
\end{tabular}

Elispot assay

Elispot testing was performed as part of the fourth CVCCRI Elispot proficiency panel [10]. All labs participating in the serum task force received five PBMC vials of each donor and five vials of each peptide pool. In addition to the same plate layout, laboratories had to follow minimal requirements to allow comparability of results, which included the total number of cells per well $(200,000)$ and the final concentration of peptide per well $(1 \mu \mathrm{g} / \mathrm{ml})$. Thus, each donor had to be tested in six replicates for reactivity against the CEF peptide pool, the CMV pp65 peptide pool, and against the appropriate medium to assess background reactivity, with an ex-vivo IFN $\gamma$ Elispot assay. Laboratories were otherwise allowed to use their own SOP including materials, reagents, and equipment. Labs performed five simultaneous experiments following the exact same SOP, but appropriately exchanged the medium for all test conditions including thawing, washing, resting and final incubation of PBMC with antigen. Viability and recovery after thawing and, if applicable after overnight resting, were recorded for each medium. Final spot counts were uploaded to the CVC data management web site. All plates were centrally re-evaluated (ZellNet Consulting, Inc., Fort Lee, NJ, USA). A response was defined as spot counts $\geq 10$ spots in antigen-stimulated wells, at least $3 \times$ above background (cells plus medium alone).

\section{Statistical analysis}

The following parameters were calculated for the overall panel and the individual participant's performance, using the central re-evaluation spot counts: the mean, standard deviation, and coefficient of variation $(\mathrm{CV})$, the median, minimum, and maximum spot counts for each donor and 
reagent and the medium only wells. Spot counts obtained from the central re-evaluation were used instead of the laboratories own spot counts in order to reduce operatordependent variability [20]. Box plots were used to illustrate the distribution of spot counts with each serum type for a given test condition. For the comparison of the spot counts between laboratories that performed an overnight rest and those that did not, a two sample Student's $t$ test was applied.

To standardize the comparison of antigen-specific spot counts for all seven positive responses across laboratories, the ranks of the spot counts were used as opposed to the actual spot count. Each laboratory generated 30 spot counts for a given donor/reagent combination $(6$ replicates $\times 5$ media). These 30 spot counts from an individual laboratory were ranked from 1 to 30 , where 1 represented the smallest spot count for that donor/reagent combination and 30 the largest. As a result, the highest average ranking possible would be 27.5 (in that case all 6 replicates of one medium would have the largest spot counts among all 30 replicates $=$ ranking $25-30$, mean $=27.5$ ), and the lowest average ranking would be 3.5 (with all replicates having the smallest spot counts compared with all other media $=$ ranking 1-6, mean 3.5). The same ranking method was also applied for the comparison of spot sizes across media and laboratories.

\section{Results}

Medium choice and antigen-specific responses

The goal of the serum task force was to identify if a serumfree medium exists that performs equally well in Elispot compared with serum-supplemented media. Among the eight donor-antigen combinations, seven positive responses could be detected, with all four donors being reactive to the CEF peptide pool, and three donors being reactive to the CMV pp65 peptide pool. Based on the reevaluation counts and prospectively established positive response definition, all laboratories detected all of these responses, when using the AIM-V, Optmizer or X-Vivo medium. Two labs did not detect the CEF response of Donor 1 with the CTL medium, due to high background reactivity. Another lab missed the CEF and CMV response of Donor 1 due to high background reactivity, but only when using lab-own medium. Figure 1 demonstrates that there is no apparent difference in spot numbers when either pretested lab-own medium/serum or serum-free media were used. Further, it can be seen that the Optmizer medium has a slightly higher mean and median spot count for most donor/antigen combinations compared to the other media. The average variability of results among all participants for a given donor/antigen/ medium combination, expressed as the percent $\mathrm{CV}$, was 39.4. In the light of different SOPs used among all groups, this comes at no surprise.

The results presented in Fig. 1 are averages across all laboratories within a media type and do not necessarily reflect the patterns occurring for an individual laboratory across the different media. To determine if the overall patterns that existed when averaging across all laboratories were consistently found within individual laboratories, a comparison was made within each laboratory between the spot counts found with each medium, using the ranking method. Thus, the larger the spot counts obtained with one medium, compared with all other media, the larger the average ranking, and vice versa. The average ranking for each medium and donor/antigen combination across all laboratories is summarized in Table 2 . The ranking results confirm that there was no apparent difference in performance between the different media and that there is a trend to higher spot counts in experiments with Optmizer medium. An interesting observation is that none of the serum-free media has an overall lower ranking than lab-own medium/serum combinations. This observation extends to results separated by donor/antigen combination, where only twice AIM-V has a slightly lower average ranking than the lab-own media. Out of all possible lab and medium/antigen combinations (not shown), 34.3\% of the largest average rankings were obtained with Optmizer medium, whereas this was the case for only $8.6 \%$ with lab-own medium.

Finally, all donor/antigen rankings were investigated for media which have the largest average ranking for an individual lab in at least 5 out of 7 possible donor-antigen combinations. Three labs were identified for which Optmizer consistently produced higher spot counts, and one lab each for AIM-V and CTL medium.

\section{Overnight resting and antigen-specific responses}

Overnight resting of thawed PBMC has been shown to positively influence spot counts $[10,12,21]$ and is one of the recommended Elispot harmonization guidelines. Since serum is believed to be essential for prolonged culture of PBMC, we investigated whether the use of serum-free media would abrogate the positive effect of a resting period before Elispot testing. Pooling the spot counts of the seven donor/antigen combinations with a positive response, a comparison was made between the spot counts from labs that did an overnight rest with those that did not. In Table 3, the average spot counts from all replicates are displayed stratified by medium type and overnight rest. A two sample $t$ test was used to compare the means of the two groups. For each medium, the mean spot count from labs that did an overnight rest was larger by more than 35 spots compared 
Fig. 1 Antigen-specific spot counts across all laboratories for each donor/antigen combination and medium. Box plots indicate the mean (triangle), median (horizontal line), 75th and 25th percentile (upper and lower box border) and minimum and maximum spot counts

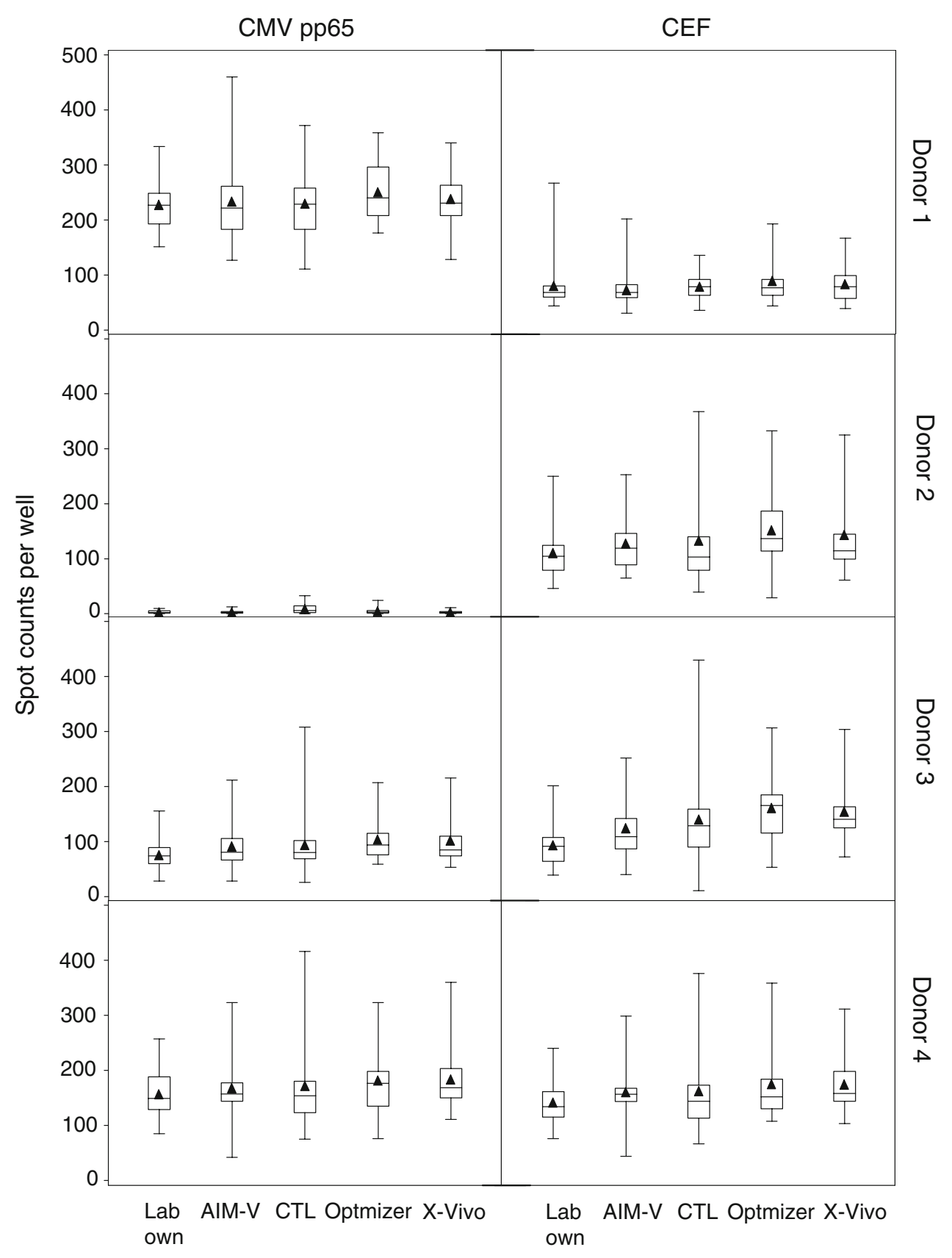

to the mean spot count from labs that did not do an overnight rest. These differences were statistically significant. Hence, serum-free conditions did not abrogate the positive effect of overnight resting.

Medium choice and background reactivity

An important aspect of medium and serum suitability for Elispot testing is the background reactivity it elicits. Figure 2 demonstrates the background reactivity level for all media across all donors. Except for a few well exceptions, background reactivity was low for all media, with a trend for the CTL medium having somewhat higher background reactivity compared to all other media. As reported earlier, the few missed responses (4 out of 385 positive responses detectable in total by all labs, across all 5 media) were caused by high background reactivity with lab-own medium (1 lab, 1 donor) or CTL medium (2 labs, 1 donor).

Medium choice and PBMC recovery and viability after thawing and overnight resting

Serum is believed to be an essential additive for optimal recovery of PBMC during the thawing process as well as for extended cell culture. On the other hand, even short 
Table 2 Overall average spot count ranking of each medium for a given donor (D)/antigen combination

\begin{tabular}{llllll}
\hline & Lab own & AIM-V & CTL & Optmizer & X-Vivo \\
\hline D1/CMV & 13.3 & 15.6 & 13.6 & 16.2 & 15.8 \\
D1/CEF & 14.0 & 13.1 & 15.9 & 15.8 & 15.3 \\
D2/CEF & 11.6 & 14.9 & 13.3 & 18.7 & 16.7 \\
D3/CMV & 11.4 & 12.0 & 13.7 & 19.0 & 18.1 \\
D3/CEF & 8.0 & 10.7 & 14.9 & 20.6 & 19.6 \\
D4/CMV & 14.5 & 12.5 & 14.1 & 17.4 & 17.1 \\
D4/CEF & 12.1 & 14.4 & 13.9 & 16.6 & 17.8 \\
Overall & 12.1 & 13.3 & 14.2 & 17.7 & 17.2 \\
\hline
\end{tabular}

exposure to serum has been noted to influence background reactivity or level of antigenic responses. Here the influence of serum-free media on the recovery and viability of cells after thawing and overnight resting was investigated. Laboratories received five separate PBMC vials from each donor batch, each of which had to be thawed in and further handled with the appropriate medium only. Eight out of 11 laboratories chose to rest cells overnight prior to the assay. One lab was excluded from this analysis due to the lack of serum supplementation for the lab-own medium. Table 4 depicts the average recovery and viability across all donors and laboratories, sorted by medium with the highest value to the medium with the lowest value. While the mean recovery after thawing appears to be slightly higher with serum-supplemented media (on average by 1.3 Million, equaling $8.6 \%$ of PBMC frozen per vial), this serum advantage disappears after overnight resting, with a similar average cell loss reported for all five media (2.9 Million), as demonstrated in Table 5. Furthermore, only minimal differences exist in the viability of cells after thawing or overnight resting among all five media.

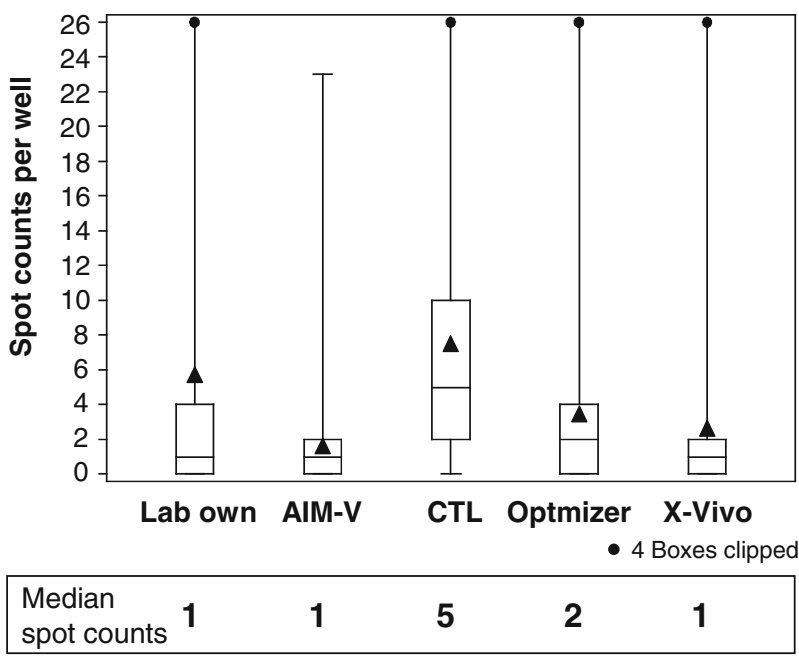

Fig. 2 Background reactivity of all media across all donors and laboratories. Spot counts were obtained by testing PBMC with medium only. Box plots indicate the mean (triangle), median (horizontal line), 75th and 25th percentile (upper and lower box border) and minimum and maximum spot counts. The figure was clipped at spot counts of 26 per well for better graphical demonstration. For each medium type there was the following number of wells that had a spot count above 26: Lab own 5 (spot counts: 105, 111, 123, 141, and 261), AIM-V 0, CTL 8 (spot counts: 27, 28, 29, 30, 30, 33, 34, and 125), Optmizer 4 (spot counts: $32,33,44$, and 60), and X-Vivo 4 (spot counts: 35,42 , 43, and 47)

\section{Medium choice and spot size}

Upon plate evaluation, multiple laboratories reported obvious spot size differences between media. To investigate whether spot size differences among plates from the same laboratory exist, spot size data were recorded during central reevaluation and further analyzed. Similar to the spot count data, spot sizes were ranked within one lab for each replicate and medium (30 rankings for 6 replicates $\times 5$ media), hence the largest average ranking possible for one medium
Table 3 Overall spot counts stratified by medium and overnight resting

\begin{tabular}{lllllll}
\hline Medium & $\begin{array}{l}\text { Overnight } \\
\text { rest }\end{array}$ & Mean & Median & $\begin{array}{l}\text { Minimum } \\
\text { spot count }\end{array}$ & $\begin{array}{l}\text { Maximum } \\
\text { spot count }\end{array}$ & $P$ value \\
\hline Lab own & Yes & 140.1 & 127 & 28 & 334 & $<0.001$ \\
& No & 101.6 & 93.5 & 38 & 219 & \\
AIM-V & Yes & 154.8 & 151 & 49 & 460 & $<0.001$ \\
& No & 107.0 & 96 & 28 & 244 & $<0.001$ \\
CTL & Yes & 156.3 & 126 & 26 & 430 & $<0.001$ \\
& No & 118.7 & 103.5 & 11 & 264 & \\
Optmizer & Yes & 178.9 & 173.5 & 44 & 359 & $<0.001$ \\
& No & 114.8 & 102.5 & 29 & 320 & 360 \\
X-Vivo & Yes & 166.7 & 154 & 39 & 268 & \\
& No & 127.4 & 109.5 & 42 & & \\
\hline
\end{tabular}


Table 4 PBMC recovery and viability stratified by medium
Rows are sorted by the mean with the media having the largest mean presented first

\begin{tabular}{llllll}
\hline Variable & Medium & Mean & Median & Minimum & Maximum \\
\hline Recovery after thawing & Lab own & 11.7 & 12.3 & 4.9 & 15.2 \\
(in Million) & Optmizer & 11.0 & 10.9 & 6.3 & 16.2 \\
& CTL & 10.5 & 10.4 & 5.3 & 16.2 \\
& X-Vivo & 10.4 & 10.3 & 5.1 & 16.4 \\
& AIM-V & 9.8 & 10.0 & 3.7 & 15.8 \\
Recovery after overnight & Lab own & 9.7 & 10.8 & 3.3 & 15.1 \\
rest (in Million) & X-Vivo & 8.8 & 9.1 & 4.0 & 13.7 \\
& Optmizer & 8.8 & 8.9 & 3.4 & 13.2 \\
& CTL & 7.8 & 8.2 & 1.6 & 12.1 \\
& AIM-V & 7.8 & 7.7 & 2.7 & 11 \\
Viability after thawing & Optmizer & 90.4 & 94.7 & 61.5 & 99 \\
(in percent) & Lab own & 90.0 & 93.0 & 56 & 99 \\
& CTL & 89.5 & 93.2 & 58 & 98.5 \\
& AIM-V & 89.3 & 93.4 & 60.4 & 99 \\
& X-Vivo & 88.7 & 89.2 & 62.8 & 99 \\
Viability after overnight & Lab own & 91.5 & 93.6 & 72.1 & 98 \\
rest (in percent) & X-Vivo & 87.5 & 89.8 & 66.7 & 97 \\
& CTL & 87.4 & 89.5 & 69.4 & 96.6 \\
& Optmizer & 86.5 & 89.9 & 57.5 & 99 \\
& AIM-V & 86.1 & 91.9 & 54.0 & 97 \\
\hline \multirow{2}{*}{} & & & & &
\end{tabular}

Table 5 Differences in cell recoveries (in Million) between thawing and overnight resting

\begin{tabular}{llllll}
\hline Serum & $N$ & Mean & Median & Minimum & Maximum \\
\hline X-Vivo & 24 & 2.2 & 2.6 & 0 & 5.9 \\
Optimizer & 24 & 2.9 & 2.6 & 0 & 8.3 \\
Lab & 24 & 2.9 & 2.7 & 0 & 6.7 \\
AIM-V & 24 & 2.9 & 2.9 & 0 & 6.9 \\
CTL & 24 & 3.5 & 3.3 & 0 & 8.8 \\
\hline
\end{tabular}

Three labs reported numbers for recovery after overnight rest that were larger than the numbers reported for recovery at thawing. This occurred for seven donor/medium combinations (AIM-V medium for donor 1, lab-own and X-Vivo medium for donor 2, CTL and X-Vivo medium for donor 3, and Optmizer and X-Vivo medium for donor 4). These differences between recoveries after thawing versus overnight rest were set to zero for these seven pairs of observations

type would be 27.5 (with all 6 replicates of one medium having the largest average spot sizes). The average ranking positions for each medium per donor/antigen combination are summarized in Table 6 . In $73 \%$ of all possible medium/ donor/antigen combinations across all laboratories, the Optmizer medium always produced highest average spot sizes, and in $87 \%$ the Optmizer medium had either the largest or second largest average spot sizes. In fact, in seven laboratories, Optmizer use resulted in largest spots in at least five out of the seven donor/antigen combinations. One lab had the largest spot sizes with the CTL medium in five out of
Table 6 Overall spot size ranking of each medium for a given donor (D)/antigen combination

\begin{tabular}{lccrll}
\hline & Lab own & AIM-V & CTL & Optmizer & X-Vivo \\
\hline D1/CMV & 12.8 & 13.7 & 12.8 & 20.5 & 13.8 \\
D1/CEF & 11.6 & 15.4 & 12.9 & 21.4 & 12.6 \\
D2/CEF & 9.7 & 15.2 & 9.4 & 23.6 & 17.2 \\
D3/CMV & 11.9 & 15.5 & 10.9 & 22.4 & 13.4 \\
D3/CEF & 12.7 & 14.7 & 12.5 & 23.0 & 11.5 \\
D4/CMV & 14.9 & 13.2 & 9.9 & 21.8 & 15.6 \\
D4/CEF & 11.7 & 11.7 & 12.2 & 22.7 & 16.4 \\
Overall & 12.2 & 14.2 & 11.5 & 22.2 & 14.3 \\
\hline
\end{tabular}

seven combinations. The remaining labs had mixed results. Figure 3 demonstrates two representative examples of the spot sizes from each of the five media within one lab for a specific donor/antigen combination.

\section{Discussion}

The presented study describes the efforts of the serum task force of the CVC-CRI to investigate whether a commercially available serum-free medium could potentially be used for replacement of serum-supplemented media in IFN $\gamma$-Elispot testing. The study originated out of recent observations in international proficiency panels that the 
Fig. 3 Spot appearance for each of the five media for the same donor/antigen and lab. Two representative examples are displayed. a Specific lab example for well B4 (Donor 1), b. b Specific lab example for well E5 (Donor 3). In both examples, spot size differences

can be recognized by eye
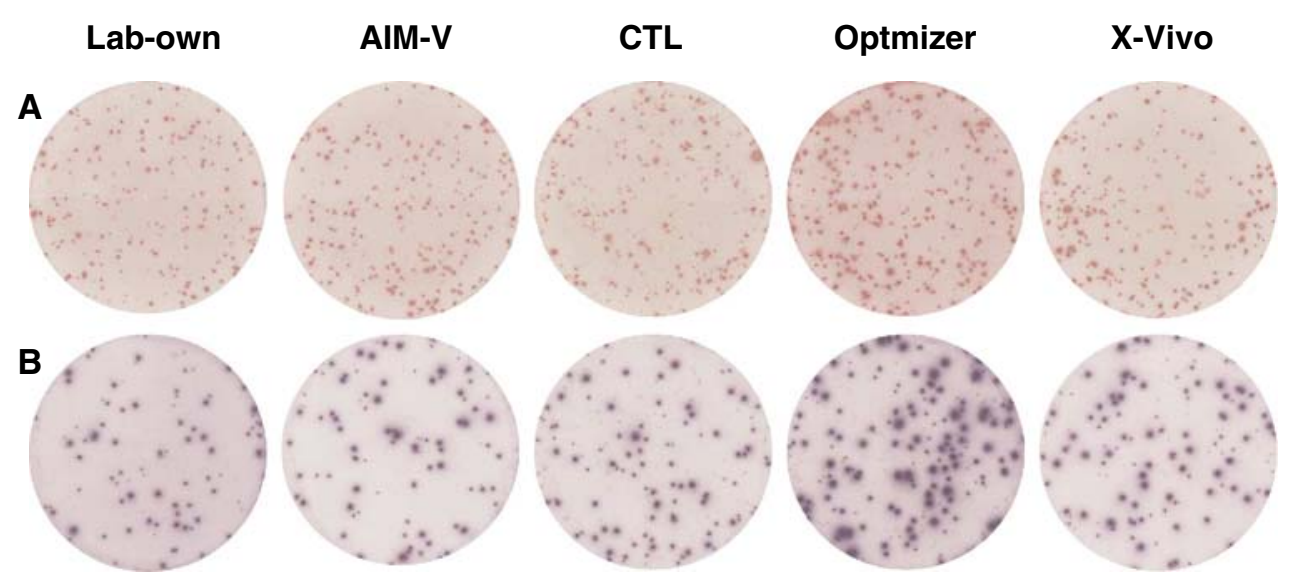

choice of serum is the leading cause for variability and suboptimal assay performance, and the knowledge that each serum batch is unique and can potentially introduce unpredictable mitogenic, stimulatory, or suppressive factors to assay conditions. A challenge for such study is the lack of a gold standard. For this reason, the serum task force was initiated composed of high-performing groups which have optimized their Elispot SOP for specific laboratory needs. Hence, each group compared serum-free media to their own medium-serum combination, and with their own unique SOP. A generalized recommendation from the serum task force is expected to hold up only if applicable across a consortium of different representative laboratories.

The excellent performance results of all serum-free media in comparison with qualified medium/serum combinations came at some surprise. The fact that no serum was added at any step of the protocol was expected to negatively influence recovery, viability and final spot counts at least for media which were not specifically developed for Elispot use like the CTL Wash and Test media. However, there were generally no apparent differences in spot counts across all media in a given laboratory. Some variability was observed, which could at least partially be accounted for by the fact that each experiment was started with a separate vial of donor PBMC, and variability could be introduced at the thawing and cell-counting step. Most interestingly, serum-free media were ranked higher in performance compared to lab-own medium/serum combinations (Table 2). These results confirm earlier observations reported by Martinuzzi [13].

An important optimization step for Elispot assays using frozen PBMC is an overnight rest during which many apoptotic cells get eliminated, resulting in a cell population with more truly viable cells and finally higher spot counts $[10,12,21]$. A critical finding from this study was that overnight resting in serum-free media does not lead to an increased cell loss in comparison to serum-supplemented media. It needs to be noted that the initial thawing process with serum-supplemented media yielded slightly higher recoveries (on average close to $9 \%$ ). This explains why slightly higher recoveries were reported after overnight resting, considering the observed similar cell loss rates as for serum-free media. Together with the well maintained spot counts and low background reactivity it can be reasonably recommended to consider the use of serum-free media in an IFN $\gamma$ Elispot, without the addition of any serum at any step of the protocol. Obviously, Standard Operating Procedures need to be optimized accordingly.

Serum-free media are highly standardized, and contain identical components in every lot, but they do not share mitogenic or suppressive factors which can potentially be found in sera. Not only do serum-free media provide a useful tool for Elispot optimization, standardization, and harmonization, but their use saves time and reduces costs since it prevents the need for pretesting of multiple serum batches and purchase of entire serum lots.

One issue not addressed in this study is the use of serum in freezing media. Short exposure to sub-optimal sera used for freezing could have unwanted effects as described earlier. Consequently, the most desirable setup would be to use serum-free freezing medium in combination with a serumfree test medium. A few serum-free freezing media are commercially available, but they have not yet been systematically investigated. To address this issue, a study is being organized by the CIMT Immunoguiding Program (C-IMTCIP) in collaboration with the CVC-CRI, and results should be expected by 2010 .

An interesting observation made was that spot sizes differed remarkably, with Optmizer spots reported to be significantly larger than any of the other media spots. The systematic analysis of spot sizes clearly confirmed this observation. It is important to stress that such changes in spot appearances require an adjustment of reading parameters for automated plate evaluation. Variation in the adjustment capabilities among the various readers and software 
versions available on the market also need to be considered, further justifying the strategy for central reevaluation of all serum task force plates.

In the majority of donor/antigen combinations Optmizer produced considerably larger spots. Since all experiments were done following the same SOP within a lab, the spot size increase indicates the secretion of more cytokine over time [4]. Given the identical experimental setup for all media within a lab including the same antigen concentration and incubation time, the Optmizer medium must provide conditions, which enhance the signal strength [22]. Such a feature could be especially beneficial for systems which are known to produce very small spots as the perforin Elispot and, therefore, require extensive optimization [23], provided that similar effects are found for cyto/chemokines other than IFN $\gamma$. For instance, the requirements to obtain optimal responses by Th2, Th17 and T-reg cells may be different, and even IFN $\gamma$ responses may vary depending on specific requirements for different ways of antigen presentation (e.g. for proteins or cell lysates). Examination of the influence of such medium on ICS results could provide further interesting results.

The results of the serum task force provide compelling evidence that serum-free media can be a safe alternative for serum-supplemented media in IFN $\gamma$ Elispot testing of PBMC. It is important to stress that each participant was a consistently high-performing laboratory that worked with a SOP that was optimized for the specific medium/serum combination routinely used. Switching to a different, serum-free medium will require optimization for the new conditions introduced. Similar conclusions have been made in the CIMT-CIP serum study [24]. The availability of serum-free medium choices provides an important tool for Elispot assay standardization and harmonization.

Acknowledgments The serum task force project was supported by the Cancer Research Institute, New York, NY, and a donation from Wyeth Vaccines, Pearl River, NY. C.M.B, C.G, and S.H.vdB were supported by the research grant "Standardization of Immune Monitoring" by the Wallace H. Coulter Foundation. G.F. was supported by the Comprehensive T Cell Vaccine Immune Monitoring Consortium (CTC-VIMC), part of the Collaboration for AIDS Vaccine Discovery with partial support from the Bill \& Melinda Gates Foundation. The authors like to thank the following persons for their active participation and support in this study: Irene Areström, Mabtech, Sweden; Mark Berrong, Duke University Medical Center, Durham, NC; Daniela Eberts and Thomas Wölfel, Johann-Gutenberg University, Department of Internal Medicine III, Mainz, Germany; Sebastian Kreiter and Petra Simon, Johann-Gutenberg University, Department of Internal Medicine III, Division of Experimental and Translational Oncology, Mainz, Germany; Waltraud Leisgang, University Hospital Erlangen, Department of Dermatology, Erlangen, Germany; Thorsten Vogel, Ben Messham and Peggy Lau, sanofi pasteur, Toronto, Canada; Marij Welters, Department of Immunohematology and Blood Transfusion, Leiden University Medical Center, Leiden, Netherlands.
Open Access This article is distributed under the terms of the Creative Commons Attribution Noncommercial License which permits any noncommercial use, distribution, and reproduction in any medium, provided the original author(s) and source are credited.

\section{References}

1. Hoos A, Parmiani G, Hege K, Sznol M, Loibner H, Eggermont A, Urba W, Blumenstein B, Sacks N, Keilholz U, Nichol G, Cancer Vaccine Clinical Trial Working Group (2007) A clinical development paradigm for cancer vaccines and related biologics. J Immunother 30:1-15

2. Chattopadhyay PK, Hogerkorp CM, Roederer M (2008) A chromatic explosion: the development and future of multiparameter flow cytometry. Immunology 125:441-449

3. Bolton DL, Roederer M (2009) Flow cytometry and the future of vaccine development. Expert Rev Vaccines 8:779-789

4. Helms T, Boehm BO, Asaad TJ, Trezza RP, Lehmann PV, TaryLehmann M (2000) Direct visualization of cytokine-producing recall antigen-specific CD4 memory T cells in healthy individuals and HIV patients. J Immunol 164:3723-3732

5. International Conference on Harmonization (ICH) (1994) Guidance for industry: Q2B validation of analytical procedures methodology. http://www.ich.org/LOB/media/MEDIA417.pdf

6. Janetzki S, Cox JH, Oden N, Ferrari G (2005) Standardization and validation issues of the ELISPOT assay. Methods Mol Biol 302:51-86

7. Mander A, Chowdhury F, Low L, Ottensmeier CH (2009) Fit for purpose? A case study: validation of immunological endpoint assays for the detection of cellular and humoral responses to antitumour DNA fusion vaccines. Cancer Immunol Immunother 58:789-800

8. NCCLS (2004) Performance of single cell immune response assays; approved guidelines. NCCLS document I/LA26-A

9. Britten CM, Janetzki S, van der Burg SH, Gouttefangeas C, Hoos A (2008) Toward the harmonization of immune monitoring in clinical trials: Quo vadis? Cancer Immunol Immunother 57:285288

10. Janetzki S, Panageas KS, Ben-Porat L, Boyer J, Britten CM, Clay TM, Kalos M, Maecker HT, Romero P, Yuan J, Kast WM, Hoos A (2008) Results and harmonization guidelines from two large-scale international Elispot proficiency panels conducted by the Cancer Vaccine Consortium (CVC/SVI). Cancer Immunol Immunother 57:303-315

11. Britten CM, Janetzki S, Ben Porat L, Clay TM, Kalos M, Maecker H, Odunsi K, Pride M, Old L, Hoos A, Romero P (2009) Harmonization guidelines for HLA-peptide multimer assays derived from results of a large scale international proficiency panel of the Cancer Vaccine Consortium. Cancer Immunol Immunother (Epub ahead of print)

12. Malyguine A, Strobl SL, Shafer-Weaver KA, Ulderich T, Troke A, Baseler M, Kwak LW, Neelapu SS (2004) A modified human ELISPOT assay to detect specific responses to primary tumor cell targets. J Transl Med 2:9

13. Martinuzzi E, Scotto M, Enée E, Brezar V, Ribeil JA, van Endert P, Mallone R (2008) Serum-free culture medium and IL-7 costimulation increase the sensitivity of ELISpot detection. J Immunol Methods 333:61-70

14. Tarte K, Fiol G, Rossi JF, Klein B (2000) Extensive characterization of dendritic cells generated in serum-free conditions: regulation of soluble antigen uptake, apoptotic tumor cell phagocytosis, chemotaxis, $T$ cell activation during maturation in vitro. Leukemia $14: 2182-2192$ 
15. Kim SJ, Diamond B (2007) Generation and maturation of bone marrow-derived DCs under serum-free conditions. J Immunol Methods 323:101-108

16. Napoletano C, Pinto D, Bellati F, Taurino F, Rahimi H, Tomao F, Panici PB, Rughetti A, Frati L, Nuti M (2007) A comparative analysis of serum and serum-free media for generation of clinical grade DCs. J Immunother 30:567-576

17. Royer PJ, Tanguy-Royer S, Ebstein F, Sapede C, Simon T, Barbieux I, Oger R, Gregoire M (2006) Culture medium and protein supplementation in the generation and maturation of dendritic cells. Scand J Immunol 63:401-409

18. Currier JR, Kuta EG, Turk E, Earhart LB, Loomis-Price L, Janetzki S, Ferrari G, Birx DL, Cox JH (2002) A panel of MHC class I restricted viral peptides for use as a quality control for vaccine trial ELISPOT assays. J Immunol Methods 260:157-172

19. Maecker HT, Dunn HS, Suni MA, Khatamzas E, Pitcher CJ, Bunde T, Persaud N, Trigona W, Fu TM, Sinclair E, Bredt BM, McCune JM, Maino VC, Kern F, Picker LJ (2001) Use of overlapping peptide mixtures as antigens for cytokine flow cytometry. J Immunol Methods 255:27-40

20. Janetzki S, Schaed S, Blachere NE, Ben-Porat L, Houghton AN, Panageas KS (2004) Evaluation of Elispot assays: influence of method and operator on variability of results. J Immunol Methods 291:175-183
21. Britten CM, Gouttefangeas C, Welters MJ, Pawelec G, Koch S, Ottensmeier C, Mander A, Walter S, Paschen A, Müller-Berghaus J, Haas I, Mackensen A, Køllgaard T, thor Straten P, Schmitt M, Giannopoulos K, Maier R, Veelken H, Bertinetti C, Konur A, Huber C, Stevanović S, Wölfel T, van der Burg SH (2008) The CIMT-monitoring panel: a two-step approach to harmonize the enumeration of antigen-specific CD8 + T lymphocytes by structural and functional assays. Cancer Immunol Immunother 57:289302

22. Hesse MD, Karulin AY, Boehm BO, Lehmann PV, TaryLehmann M (2001) A T cell clone's avidity is a function of its activation state. J Immunol 167:1353-1361

23. Zuber B, Levitsky V, Jönsson G, Paulie S, Samarina A, Grundström S, Metkar S, Norell H, Callender GG, Froelich C, Ahlborg N (2005) Detection of human perforin by ELISpot and ELISA: ex vivo identification of virus-specific cells. J Immunol Methods 302:13-25

24. Mander A, Gouttefangeas C, Ottensmeier C, Welters M, Low L, van der Burg SH, Britten CM, for the ELISPOT proficiency panel of the CIMT Immunoguiding Program (2009) Should serum be used in the ELISPOT assay? A direct comparison of sixteen representative IFN- $\gamma$ ELISPOT protocols. Cancer Immunol Immunother (submitted) 